\title{
VACuum INtubation (VACcIN) box restricts the exhaled air dispersion generated by simulated cough: description and simulation-based tests of an innovative aerosolization protective prototype
}

\author{
Rami Issa $^{1} \cdot$ Robert Urbanowicz ${ }^{1} \cdot$ Philippe Richebé $^{1} \cdot J^{\prime}$ ulie Blain ${ }^{1} \cdot$ Alexandre Ferreira Benevides $^{2} \cdot$ Issam Tanoubi $^{1}$ (D)
}

Received: 5 January 2021 / Accepted: 16 September 2021 / Published online: 25 September 2021

(c) The Author(s), under exclusive licence to Springer Nature B.V. 2021

\begin{abstract}
The COVID-19 pandemic has caused personal protective equipment shortages worldwide and required healthcare workers to develop novel ways of protecting themselves. Anesthesiologists in particular are exposed to increased risks of contamination when performing interventions such as airway manipulations. We developed and tested an aerosolization protective device which contains aerosols around the patient's airway and helps eliminate particles using negative pressure. This intubation box is a polymethyl methacrylate prism with openings for gloves, integrated suction and ventilation connectors. We conducted a randomised controlled series of tests to detect $0.5 \mu \mathrm{m}$ particles after a simulated cough inside the intubation box, using a high-fidelity simulation mannequin. Setting and main outcome: We measured particle concentrations inside the box with and without suction turned on, in both negative and positive pressure operating rooms. We also obtained particle concentrations outside our box and compared them to non-airtight barrier devices. One minute following simulated cough, the mean number of particles per cubic foot in our box with suction on is around $45 \%$ that with the suction off $(1,462,373 \mathrm{vs}$ $3,272,080, \mathrm{P}<0.0001)$ in the negative pressure room, and four times lower than with the suction off $(760,380 \mathrm{vs} 3,088,700$, $\mathrm{P}<0.0001$ ) in the positive pressure room. After a simulated cough inside the box, particles can be detected in front of the anesthesiologist's face with a non-airtight device, while none are detected when our box is sealed and its suction turned on. The use of our negative pressure intubation box prevents contamination of surroundings and increases particle elimination, regardless of room pressure.
\end{abstract}

Keywords COVID-19 · Aerosols · Particles · Intubation box

\section{Introduction}

At the beginning of the COVID-19 (SARS-CoV-2) pandemic, a shortage of personal protective equipment forced healthcare workers around the world to be inventive and to develop novel ways to protect themselves [1,2]. While that shortage has been resolved in most countries, it could remain an issue in developing countries and the threat of variants may once more test our ability to maintain an adequate

Issam Tanoubi

i.tanoubi@umontreal.ca

1 Department of Anesthesiology and Pain Medicine of Maisonneuve-Rosemont Hospital, CIUSSS de l'Est de l'Ile de Montreal, University of Montreal, 5415 Boulevard de l'Assomption, Montreal, QC H1T 2M4, Canada

2 Department of Mechanical Engineering, Ecole Polytechnique de Montréal, Montreal, QC, Canada supply of PPE. Of concern is the potential for viral airborne transmission by way of aerosols generated during a patient's respiratory activity or secondary to medical procedures such as intubation and extubation [3]. Early in the advent of the coronavirus pandemic, multiple intubation boxes were designed as a protective barrier to help diminish the spread of aerosols. However, much emphasis has been put on the physical separation of the patient and the healthcare worker and not on the elimination of the aerosols present in the vicinity of the patient [4]. Furthermore, there is ongoing debate as to whether these intubation boxes serve their purpose as a mean of shielding medical staff from infectious droplets, and a recent study even suggested they increased intubation difficulty [5]. Finally, there is no standardized test to determine the efficacy of these intubation boxes at eliminating aerosols.

The aim of this project was to develop and test an aerosolization protective device, the VACuum INtubation 
(VACcIN) Box, that not only serves as a physical barrier to aerosols, but also creates a negative pressure environment which helps eliminate aerosols altogether. The main objective of the simulation-based tests with mannequin was to measure particle concentration after a cough inside and outside the box in several settings.

\section{Materials and methods}

\subsection{Ethics}

Ethical approval for this study was not required as the study was carried out on a high-fidelity simulation mannequin, did not involve any risk for the investigators and did not disturb the patient's access to the operating room. We also ensure to use operating equipment that could not be needed for the patient.

\subsection{Technical design}

The design of the box began in March 2020 and the experiments took place from May to July 2020. With the help of engineers and designers from École Polytechnique de Montréal and Santé Libre-a non-profit organization-and thanks to the expertise of the PI and CoPI (first and second author) in bioengineering, we have developed an intubation device which meets the following requirements: low-cost assembly, simple and intuitive design, comfortable for the user, reusable, minimum number of parts, easy to clean and decontaminate, and adaptable to various settings such as the operating room (OR), emergency room (ER) and intensive care unit (ICU).

Our device is a modified rectangular prism made from polymethyl methacrylate (PMMA). Its dimensions are $620 \mathrm{~mm}(\mathrm{~W}) \times 460 \mathrm{~mm}(\mathrm{H}) \times 400 \mathrm{~mm}(\mathrm{D})$. We have incorporated in the box four essential elements (Fig. 1):

1. Four circular openings (two on the front side and two on the right side of the box, diameter $135 \mathrm{~mm}$ ) with ridges. These allow the attachment of single-use XL nitrile gloves with 16" cuffs in an airtight manner. The gloves can be fitted onto the box's four openings and provide protected access to the patient to up to two operators. The two lateral openings could also be used to pass cables or other instruments.

2. Suction inlet (High-Density Polyethylene) that continually aspirates the air inside the box. This connects to a standard hospital medical aspiration wall port via single use corrugated plastic tubing and an adaptor to a rigid aspiration tube. It is thus usable in most hospital areas where intubations occur. This suction inlet defines the main characteristic of the VACuum INtubation (VAC-
cIN) Box, namely a continuous negative pressure and therefore a vacuum effect inside.

3. Integrated ventilation inlet (High-Density Polyethylene) which saddles the lateral panel of the box. This ventilation adaptor is screwed onto the PMMA panel with an O-ring to achieve airtightness. It can be removed and decontaminated after each use. The adaptor presents a female connector on the inside of the box that connects directly to the endotracheal tube, and a male connector on the outside of the box that connects to the ventilator circuit. Therefore, the patient can be ventilated through this adaptor without having to remove the box.

4. Opening to accommodate the patient's thorax, including a notched arch to allow the placement of a single-use transparent adhesive polyurethane film (Opsite ${ }^{\diamond}$ Incise: https://www.smith-nephew.com/fr-canada/produits/ traitement-avance-des-plaies/opsite--champ---incis er/, Ontario, Canada) to seal the gaps between the box and the patient's torso (opening width at base $440 \mathrm{~mm}$, center height $330 \mathrm{~mm}$ ).

\subsection{Testing and data}

In a real operating room (OR), we performed three sets of tests in mannequin with the VACcIN Box and a simulated patient cough (Fig. 2):

1. In a negative pressure $\mathrm{OR}$, measurement of particles inside the box with and without suction (Fig. 2)

2. In a positive pressure $\mathrm{OR}$, measurement of particles inside the box with and without suction (Fig. 2)

3. In a negative pressure OR, measurement of particles outside the box, in front of the anesthesiologist in three situations (Fig. 3):

a. No box

b. VACcIN box used as a physical shield to separate the patient from the anesthesiologist, with all holes unsealed and no suction

c. VACcIN box with all holes sealed and with suction

To quantify the effect of the suction connector on particle elimination, we devised a simple, low-cost test using local operating room equipment. In a negative pressure OR, a simulation mannequin (SimMan 3G, https://www.laerd al.com/ca/products/simulation-training/emergency-caretrauma/simman-3g/) was positioned with its head inside the box and all equipment (connectors, gloves, surgical film) was installed as described previously (Fig. 2). Cough aerosol particles typically have diameters under $1 \mu \mathrm{m}$, an average closer to $0.5 \mu \mathrm{m}$ [6]. A TPI@ 1020 Handheld Particle Counter (https://www.itm.com/fr/produit/tpi-1020-handheld-parti 


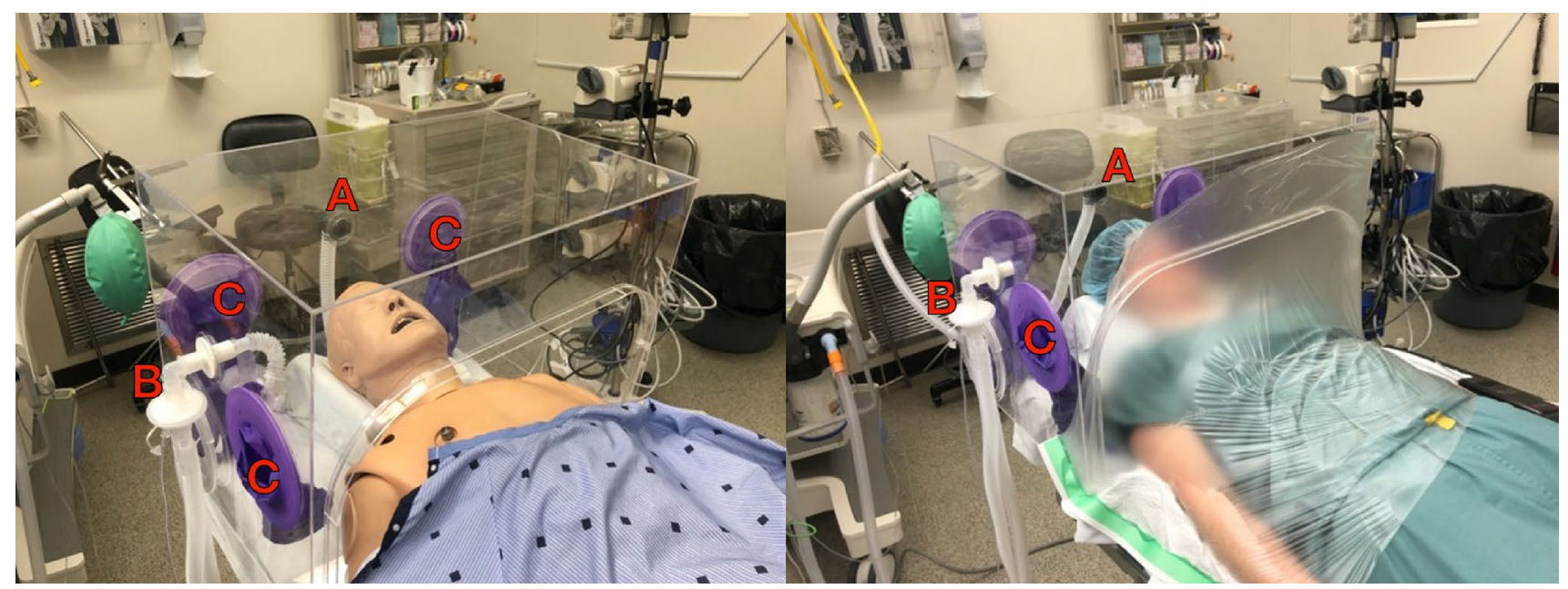

Fig. 1 Setup used for particle measurements with mannequin (left) and with a healthy volunteer (right), showing connected medical vacuum (A) and ventilator circuits (B), and threee purple nitrile gloves attached on their dedicated openings (C). (A) Suction inlet (HighDensity Polyethylene) that continually aspirates the air inside the box, connected to a standard hospital medical vacuum wall port. (B) Ventilation inlet (High-Density Polyethylene) which saddles the lateral panel of the box, screwed onto the polymethyl methacrylate (PMMA) panel with an O-ring to achieve airtightness. The adaptor presents a female connector on the inside of the box that connects directly to the endotracheal tube, and a male connector on the outside of the box that connects to the ventilator circuit. (C) Four circular openings that allow the attachment of nitrile gloves with 16" cuffs in an airtight manner. The two lateral openings could also be used to pass cables or other instruments

1. Mannequin lying on surgical table without the box

2. Mannequin lying on surgical table, box in place (physical shield), but with gloves removed and all holes unsealed

3. Mannequin lying on surgical table, box in place (VACcIN box), all holes sealed with respective equipment and suction $O N$

This allows us to determine whether $0.5 \mu \mathrm{m}$ particles generated by a cough simulation can be detected adjacent to an anesthesiologist's airway, presumably mimicking a transmission mechanism. This test would also indicate whether an imperfect physical barrier-such as the many intubation boxes designed in the previous months - prevents these particles from spreading. Finally, it would show whether our box's design characteristics potentially provide additional protection.

\subsection{Statistics}

The primary endpoint, the particle concentration (number of particles per cubic foot), during the first minute after the simulated cough, was tested with a normal distribution (D'Agostino \& Pearson test). To compare the primary endpoint between VACcIN box group and Control group we used the repeated measures two-way ANOVA test with Sidak's multiple comparisons test. The number of particles was expressed as mean (standard deviation SD). We used the results (mean (SD)) of five preliminary tests performed anesthesiologist in the following 3 situations (Fig. 3): 

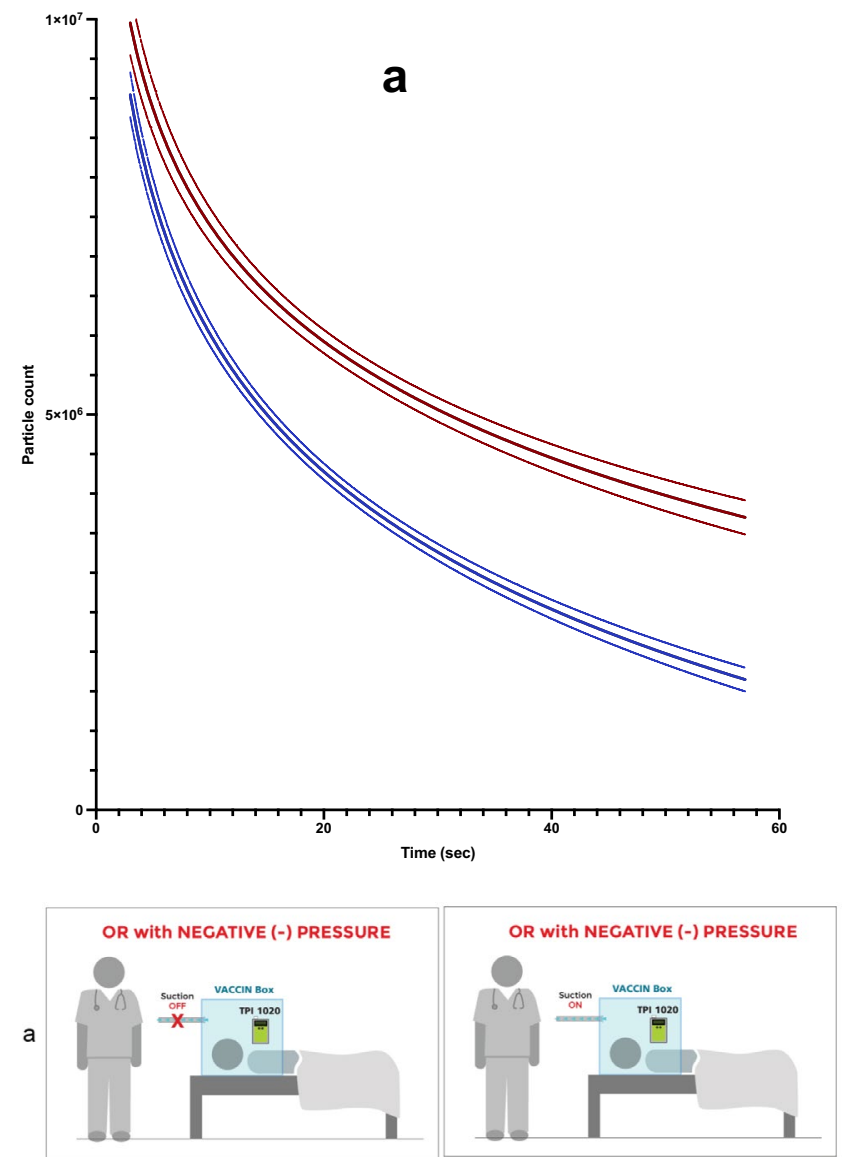

Fig. 2 Measurement of particles inside the box: schematics of the sets of tests and results. a Interpolated particle concentration (mean number of particles per cubic foot, $95 \% \mathrm{CI}$ ) inside VACcIN box with (blue) and without (red) applied suction, $60 \mathrm{~s}$ following simulated

using the protection box without a negative pressure to calculate the number of tests (sample size) needed to demonstrate a $25 \%$ reduction of the particle count inside the box at the end of the test. Relying on Bernard Rosner's method (https://www.stat.ubc.ca/ rollin/stats/ssize/n2.html), we found that 10 tests per group were needed (power: 0.80; type I error: 0.05). For our first set of tests, we chose to perform 15 measures in each group to compensate for a data loss. After we performed the first set of tests, we found that there was no data loss, and thus performed only 10 tests for the following sets as calculated.

\section{Results}

Within the negative pressure operating room, we performed 15 tests with the VACcIN box (suction on) and 15 with the control box (suction off) without data loss. The pressure in the VACcIN box was maintained negative all through the test. The particle measurement was carried out
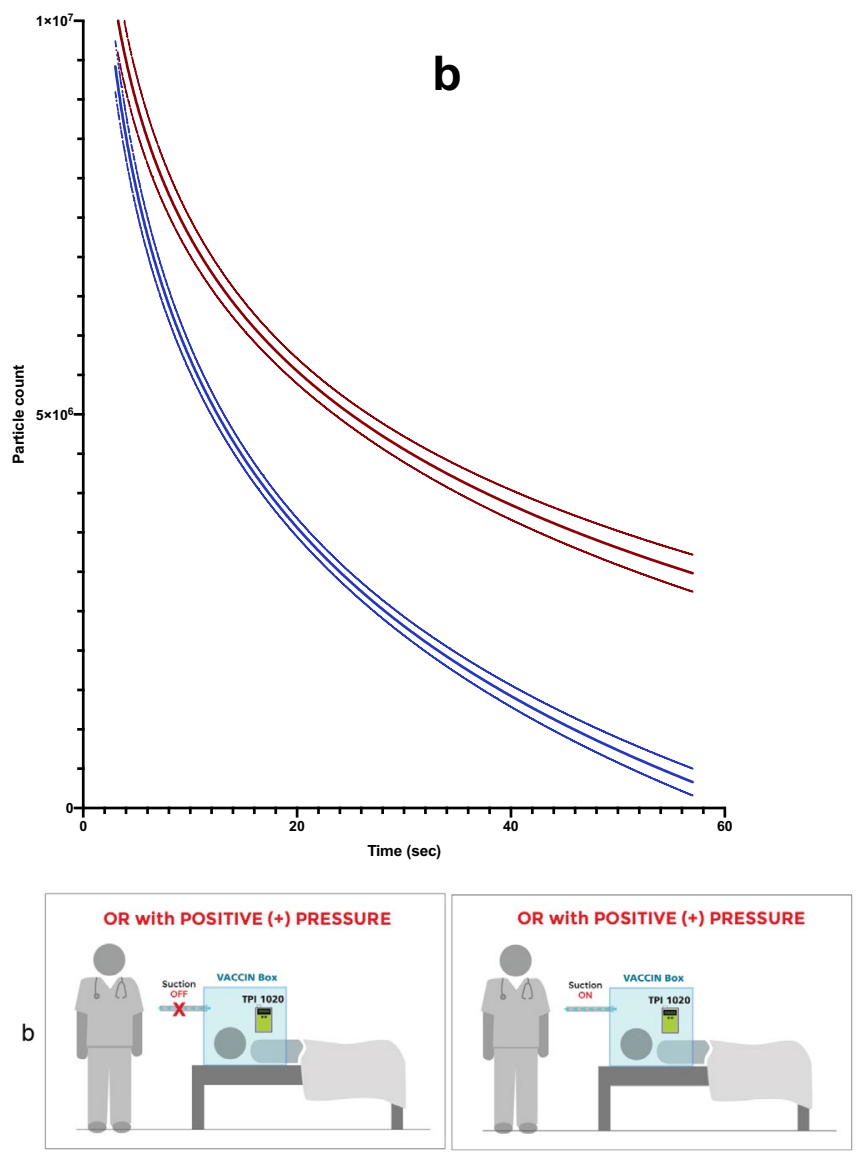

cough, in negative pressure OR. b Interpolated particle concentration (mean number of particles per cubic foot, 95\% CI) inside VACcIN Box with (blue) and without (red) applied suction, $60 \mathrm{~s}$ following simulated cough, in positive pressure OR

inside the box. The VACcIN box exhibited significantly fewer particle counts during each $3 \mathrm{~s}$ of the test (Two-way RM ANOVA of the percentage of total variation $\times$ Time, $\mathrm{F}(18,266)=1.908, \mathrm{P}=0.0156$, Fig. 2). The difference in the number of particles between the groups became significant as early as the 9th second after the cough. The mean number of particles was halved at the 21 st second in the VACcIN box and at the 39th second in the control group. After 1 min, the mean concentration of particles (number of particles per cubic foot) in the VACcIN box is around $45 \%$ that of the control group $(1,462,373$ vs $3,272,080$, $\mathrm{t}=5.990, \mathrm{DF}=266, \mathrm{P}<0.0001)$.

Within the positive pressure operating room, we performed 10 tests with the VACcIN box (suction on) and 10 with the control box (suction off) without data loss. The pressure in the VACcIN box was maintained negative all through the test. The particle measurement was carried out inside the box. The VACcIN box exhibited significantly fewer particle counts during each $3 \mathrm{~s}$ of the test (Two-way RM ANOVA of the percentage of total variation $\times$ Time, $\mathrm{F}(18,171)=1.908, \mathrm{P}=0.0007$, Fig. 2$)$. The difference in 

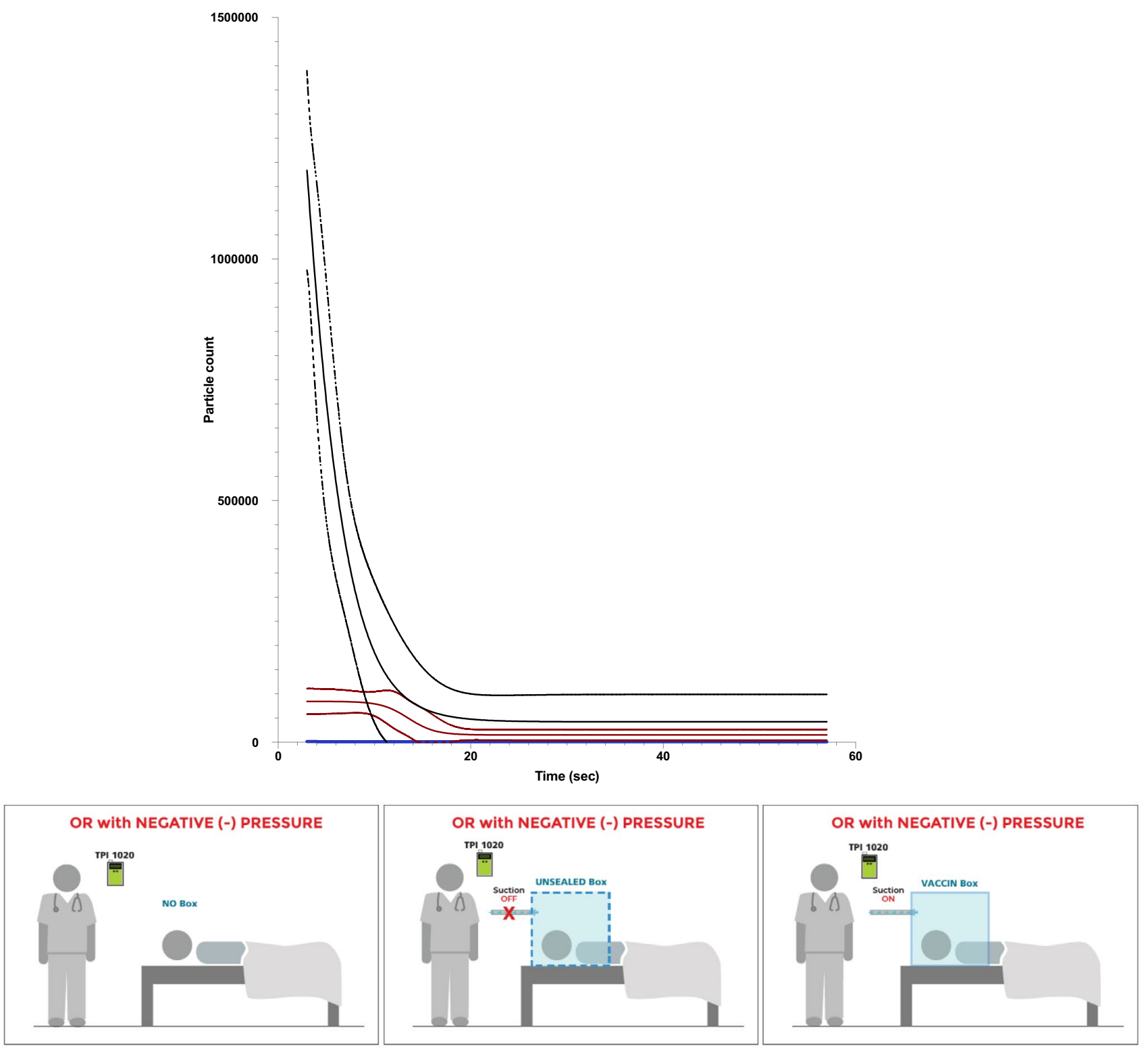

Fig. 3 Measurement of particles outside the box: schematics of the sets of tests and results: interpolated particle concentration (mean number of particles per cubic foot, $95 \% \mathrm{CI}$ ) measured in front of the face of a mask-wearing anesthesiologist in three situations: no box (black), unsealed box (red), VACcIN box sealed, and suction applied (blue), $60 \mathrm{~s}$ following simulated cough, in negative pressure OR the number of particles between the groups became significant as early as the 9th second after the cough. The mean number of particles was halved at the 15th second in the VACcIN box and at the 45th second in the control group. After 1 min, the mean number of particles in the VACcIN box is more than four times lower than in the control group $(760,380$ vs $3,088,700, t=6,286, \mathrm{DF}=171, \mathrm{P}<0.0001)$.

Finally, the measurements made in the 3 situations where the TPI@ 1020 Handheld Particle Counter is in front of the anesthesiologist's airway (outside the box) revealed that $0.5 \mu \mathrm{m}$ particles can be detected without the box or with the unsealed box. However, no particles are detected if all glove and connector openings are sealed, and suction is applied to the box (Two-way RM ANOVA of the percentage of total variation $\times$ Time, $\mathrm{F}(36,342)=6.163$, $\mathrm{P}<0.0001$ (Fig. 3). 


\section{Discussion}

The aim of this study was to develop and test an aerosolization protective device that serves as a physical barrier to aerosols with the added value of a negative pressure environment that eliminates aerosols in potentially infectious patients.

Our study quantifies the number of particles inside an aerosol protective device after a simulated cough. Previous studies have examined the particle dispersion inside such devices with only qualitative data [4-7]. Our study has shown that there is a $30 \%$ reduction of $0.5 \mu \mathrm{m}$ particle count inside the VACcIN box as early as the 9th second after a simulated cough when a negative pressure is applied inside the box, in a negative pressure OR. Furthermore, this trend progresses as there is a 55\% reduction of particle count inside the box after 1 min of suction. Similar tests in a positive pressure OR also show a significant decrease of particle count at the 9th second, but with a greater reduction of $75 \%$ after $1 \mathrm{~min}$. The improved particle elimination in a positive pressure OR could be explained by an increased pressure gradient between the outside of the box and the suction inlet. All these results demonstrate the added value of having a continuous suction placed inside the box. Our study showed that particles are undetectable outside the box when the suction is on, with the current measurement systems you use, however, patients should be intubated as soon as conditions are favorable, with the knowledge that infectious particles are being actively removed by the box.

Our data also suggests that an aerosolization protective device without particle aspiration or adequate seal is unable to prevent aerosols from leaving the vicinity of the patient and potentially contaminating OR staff and environment. This was made evident by the presence of $0.5 \mu \mathrm{m}$ particles near the operator's face up to a minute after simulated cough with no box or when an unsealed box was used. Furthermore, the use of negative pressure in the VACcIN box grants it a potential advantage over other physical barriers: that of reducing particle dispersion upon its removal.

If properly implemented, the use of a negative pressure intubation box could provide an alternative to the use of personal protective equipment when these are in short supply. In institutions where PPE is available, it could serve as additional protection to reduce the risk of contamination for medical staff. The option and ability to ventilate an apneic patient within the box seems like a potential benefit to managing patients with severe respiratory failure. Finally, with further studies aimed to determine the particle count threshold at which OR contamination is deemed improbable, the effect of a negative pressure box could decrease OR downtime. Such a device could render the waiting time for proper operating room air-cycling unnecessary in patients suspected of or confirmed as COVID-19 positive.

The VACcIN box is certainly responding to the current pandemic, but its use could easily be redesigned for other patients who could expose healthcare staff to airborne droplets disease.

This study certainly has limitations. Salbutamol inhalers do generate a wide range of particle sizes with an average of $1 \mu \mathrm{m}$ [8], and most of these are between 0.5 and $5 \mu \mathrm{m}$ [9]. Particles under $1 \mu \mathrm{m}$ in size are present to a lesser extent, but there are clearly enough to be detected by the TPI $\odot 1020$ we used. We chose to measure $0.5 \mu \mathrm{m}$ particles because we wanted to simulate a scenario where aerosols diffused widely, were more likely to escape the prototype box and thus contaminate the OR and its occupants. Our results may not be applicable to particles finer than $0.5 \mu \mathrm{m}$, as these may behave differently from their larger counterparts, and thus may not fit the model presented here.

Three puffs of salbutamol were used to simulate patient cough. These probably underestimate the coughing spells sometimes seen when manipulating a patient's airway. For that reason, it may be interesting to test the effectiveness of the VACcIN Box in more forceful coughing situations. Furthermore, it is important to remember that the TPI $\odot 1020$ was placed in the center of the box and its recordings reflect particle concentrations at that specific location. Our study provides no information as to the distribution of particles inside the box. There are likely to be areas where the effect of the suction is minimal because they are outside the path of airflow from the patient's torso to the suction inlet. In these zones, particles could stagnate and therefore not be eliminated. The box's corners are likely to be susceptible to such a phenomenon. Finally, we may ask ourselves whether the seal achieved with the adhesive surgical drape on the mannequin is equivalent to what could be obtained with an actual patient.

Further study is necessary to determine the particle count threshold inside the VACcIN Box at which outside contamination is deemed improbable, to safely remove the VACcIN Box to position the patient after induction. We demonstrated the value of creating a negative pressure environment besides a physical barrier surrounding intubation, being an aerosol-generating procedure at risk of COVID-19 transmission. Our goal is also to increase knowledge about intubation boxes aiming to diminish coronavirus contamination and transmission. However, further research should establish the safety and ease of airway management before its use on COVID-19 positive patients [10].

In conclusion, this study demonstrates that the VACcIN intubation box, placed tightly without leaks around the head and the patient's upper chest and equipped with negative 
pressure, prevents contamination of surroundings and increases particle elimination, regardless of room pressure.

Acknowledgements The authors would like to thank the department of anesthesiology and pain medicine of the Maisonneuve-Rosemont hospital for their support.

Funding Support was provided solely from institutional and/or departmental sources.

\section{Declarations}

Conflict of interest The authors declare that they have no conflict of interest.

Ethical approval Not applicable.

Consent to participate Not applicable.

\section{References}

1. Meng L, Qiu H, Wan L, et al. Intubation and ventilation amid the COVID-19 outbreak: Wuhan's experience. Anesthesiology. 2020;132(6):1317-32. https://doi.org/10.1097/ALN.0000000000 003296.

2. Institut National de Santé Publique (INSPQ) Mesures exceptionnelles pour les équipements de protection individuelle lors de pandémie: recommandation intérimaires 2020. https://www.inspq.qc. $\mathrm{ca} /$ sites/default/files/covid/2957-mesures-exceptionnelles-equip ements-protection-individuelle-covid19.pdf. Accessed on 31 Aug 2021.

3. Peng PWH, Ho PL, Hota SS. Outbreak of a new coronavirus: what anaesthetists should know. Br J Anaesth. 2020;124(5):497-501. https://doi.org/10.1016/j.bja.2020.02.008.
4. Canelli R, Connor CW, Gonzalez M, Nozari A, Ortega R. Barrier enclosure during endotracheal intubation. N Engl J Med. 2020. https://doi.org/10.1056/NEJMc2007589.

5. Begley JL, Lavery KE, Nickson CP, Brewster DJ. The aerosol box for intubation in coronavirus disease 2019 patients: an in-situ simulation crossover study. Anaesthesia. 2020. https://doi.org/10. 1111/anae.15115.

6. Lindsley WG, Pearce TA, Hudnall JB, et al. Quantity and size distribution of cough-generated aerosol particles produced by influenza patients during and after illness. J Occup Environ Hyg. 2012;9(7):443-9. https://doi.org/10.1080/15459624.2012.684582.

7. Matava CT, Yu J, Denning S. Clear plastic drapes may be effective at limiting aerosolization and droplet spray during extubation: implications for COVID-19. Can J Anaesth. 2020;67(7):902-4. https://doi.org/10.1007/s12630-020-01649-w.

8. Taylor RH, Lerman J, Chambers C, Dolovich M. Dosing efficiency and particle-size characteristics of pressurized metered-dose inhaler aerosols in narrow catheters. Chest. 1993;103(3):920-4. https://doi.org/10.1378/chest.103.3.920.

9. Crim C, Holmes M, Lee B, Cavanaugh R, Lincourt W. Evaluation of particle size distribution of albuterol sulfate hydrofluoroalkane administered via metered-dose inhaler with and without valved holding chambers. Ann Allergy Asthma Immunol. 2005;94(1):80 5. https://doi.org/10.1016/S1081-1206(10)61290-2.

10. Cook TM, El-Boghdadly K, McGuire B, McNarry AF, Patel A, Higgs A. Consensus guidelines for managing the airway in patients with COVID-19: guidelines from the Difficult Airway Society, the Association of Anaesthetists the Intensive Care Society, the Faculty of Intensive Care Medicine and the Royal College of Anaesthetists. Anaesthesia. 2020;75(6):785-99. https://doi.org/ 10.1111/anae.15054.

Publisher's Note Springer Nature remains neutral with regard to jurisdictional claims in published maps and institutional affiliations. 